\title{
Combination treatment with trimetazidine and diltiazem in stable angina pectoris
}

\author{
S C Manchanda, S Krishnaswami
}

\begin{abstract}
Objective-To assess antianginal efficacy and possible adverse haemodynamic effects of combination treatment with trimetazidine and diltiazem in patients with stable angina.

Design-Double blind, randomised, pla-
\end{abstract} cebo controlled trial of four weeks duration.

Setting-Outpatient department of two Indian hospitals.

Subjects-64 male patients with stable angina, uncontrolled on diltiazem alone. Interventions-Diltiazem $180 \mathrm{mg}$ and trimetazidine $60 \mathrm{mg}$, or diltiazem $180 \mathrm{mg}$ and placebo daily.

Main outcome measure-Change in exercise time to $1 \mathrm{~mm} \mathrm{ST} \mathrm{segment} \mathrm{depression.}$ Results-33 patients (55\%) had no exercise induced angina at $3 \mathrm{~mm}$ ST segment depression at inclusion in the study (silent ischaemia). Intention to treat analysis showed that of 32 patients in each treatment group, the number (\%) of patients responding to trimetazidine compared to placebo was: for anginal attacks, $28(87.5)$ $v 15$ (46.9), p < 0.001 ; for exercise time to 1 mm ST segment depression, 21 (65.6) $v 9$ (28.1), p $<0.003$; for exercise time to angina, $12(37.5) v 5$ (15.6), $p<0.05$; and for maximum work at peak exercise, 17 (53.1) $v 8$ (25), $\mathrm{p}<0.02$. Compared to placebo, there was net improvement with trimetazidine in mean anginal attacks of 4.81 week (95\% confidence interval (CI) 7.5 to $2.1 ; p<0.002)$; in mean exercise times at 1 mm ST segment depression of 94.2 seconds (95\% CI 182.8 to $5.6 ; p<0.05)$, and at onset of angina of 113.1 seconds (95\% CI 181.6 to 44.6 ; $p<0.02$ ); and in mean maximum work at peak exercise of 1.4 metabolic equivalents (95\% CI 2.4 to 0.3 ; p < 0.05).

Conclusions-Patients with stable angina uncontrolled with diltiazem had a clinically important improvement after combination treatment with trimetazidine, without adverse haemodynamic events or increased side effects.

(Heart 1997;78:353-357)

Keywords: trimetazidine; diltiazem; blood pressure; stable angina; treatment

Dr Manchanda, Department of Cardiology, All India Institute of Medical Sciences, Ansari Nagar, New Delhi 110029 , India.

Accepted for publication 24 June 1997

Patients with stable angina pectoris are often effectively controlled by monotherapy with nitrates, $\beta$ blockers, or calcium entry blockers. When symptoms intensify, these drugs are used in combination. However, there are problems with their use because of adverse effects, contraindications, and the fact that they are not always effective, ${ }^{1}$ and even triple drug therapy may not confer a clear clinical advantage. ${ }^{2}$ Alternative treatment with coronary artery bypass grafting (CABG) and percutaneous transluminal coronary angioplasty (PTCA) is effective, but up to $28 \%$ of patients undergoing CABG, and $75 \%$ undergoing PTCA, require at least one antianginal drug after six months. ${ }^{3}$ Further, these procedures are unaffordable or inaccessible for most patients in developing countries like India, where an estimated 40 million people suffer from coronary artery disease. ${ }^{4}$ There is therefore a continuing need for effective, safe, and acceptable antianginal drug treatment.

Trimetazidine-1-(2,3,4 trimethoxybenzyl)piperazine dihydrochloride (Flavedon 20) - is a lesser known member of a new class of antianginal drugs, ${ }^{5}$ although it has been used in many Asian and European countries for several years. Its mode of action is different from $\beta$ blockers, calcium entry blockers, and nitrates. Unlike these antianginal agents, which affect haemodynamic determinants of the myocardial oxygen supply-demand balance, ${ }^{1}$ trimetazidine partly prevents intracellular metabolic changes such as depletion of adenosine triphosphate (ATP) and phosphocreatine, accumulation of protons, and toxic free radical generation which result from ischaemia and reperfusion in the myocardium. ${ }^{6}$

In controlled studies, the antianginal efficacy of trimetazidine in monotherapy was greater than placebo, ${ }^{7}$ and equal to nifedipine, ${ }^{8}$ or propranolol, ${ }^{9}$ with no effect on heart rate and blood pressure and no major adverse effects. ${ }^{7}$ Diltiazem, a calcium entry blocker with confirmed efficacy in the treatment of stable angina, increases coronary blood flow and reduces myocardial oxygen demand by peripheral arterial vasodilatation and a negative inotropic effect. ${ }^{1}$ The action of trimetazidine could enhance the antianginal efficacy of diltiazem when the two are used in combination, without increasing adverse haemodynamic effects such as reflex tachycardia, bradycardia, hypotension, or fatigue that occur with other drug combinations (such as $\beta$ blockers with calcium entry blockers or nitrates). ${ }^{1}$

In this two centre, double blind, randomised, placebo controlled trial, we assessed combination treatment with trimetazidine and diltiazem for patients with stable angina. 
Table 1 Baseline characteristics of patients according to study group

\begin{tabular}{|c|c|c|}
\hline Characteristic & $\begin{array}{l}\text { Trimetazidine and } \\
\text { diltiazem }(n=32)\end{array}$ & $\begin{array}{l}\text { Placebo and } \\
\text { diltiazem }(n=32)\end{array}$ \\
\hline Age (years $(\mathrm{SD})$ ) & $56.6(7.5)$ & $55.0(5.0)$ \\
\hline Male sex $(\%)$ & $32(100)$ & $32(100)$ \\
\hline Indian race $(\%)$ & $32(100)$ & $32(100)$ \\
\hline \multicolumn{3}{|l|}{ NYHA functional class* } \\
\hline $2(\%)$ & $21(65.6)$ & $23(71.9)$ \\
\hline $3(\%)$ & $11(34.4)$ & $9(28.1)$ \\
\hline Duration of stable angina (months (SD)) & $39.0(37.1)$ & $27.1(24.0)$ \\
\hline Patients with silent ischaemia $\nmid(\%)$ & $16(50)$ & $8(25)$ \\
\hline History of myocardial infarction $\ddagger(\%)$ & $8(25)$ & $8(25)$ \\
\hline \multicolumn{3}{|l|}{ Previous treatment for angina } \\
\hline Percutaneous transluminal coronary angioplasty (\%) & $2(6.3)$ & $0(0)$ \\
\hline Long acting nitrates $(\%)$ & $14(43.7)$ & $15(46.9)$ \\
\hline$\beta$ Blockers (\%) & $26(81.3)$ & $25(78.1)$ \\
\hline Calcium channel antagonists $(\%)$ & $19(59.4)$ & $15(46.9)$ \\
\hline Two drug combination (\%) & $16(50)$ & $19(59.4)$ \\
\hline Three drug combination (\%) & $5(15.6)$ & $5(15.6)$ \\
\hline Resting heart rate (per min (SD)) & $84.0(13.8)$ & $80.2(12.5)$ \\
\hline Systolic blood pressure $(\mathrm{mm} \mathrm{Hg}(\mathrm{SD}))$ & $139.4(18.1)$ & $136.8(17.2)$ \\
\hline Diastolic blood pressure ( $\mathrm{mm} \mathrm{Hg}(\mathrm{SD}))$ & $85.5(10.9)$ & $85.3(8.8)$ \\
\hline Total cholesterol $(\mathrm{mmol} / \mathrm{l}(\mathrm{SD}))$ & $5.47(0.81)$ & $5.83(1.28)$ \\
\hline HDL cholesterol (mmol/l (SD)) & $1.00(0.13)$ & $1.01(0.15)$ \\
\hline Total triglyceride (mmol/1 (SD)) & $1.97(0.92)$ & $1.98(0.75)$ \\
\hline Fasting blood glucose (mmol/1 (SD)) & $5.52(1.27)$ & $5.78(1.49)$ \\
\hline Serum creatinine $(\mu \mathrm{mol} / 1(\mathrm{SD}))$ & $88.4(8.8)$ & $79.6(8.8)$ \\
\hline
\end{tabular}

HDL, high density lipoprotein.

${ }^{\star}$ New York Heart Association classification. ${ }^{10}$

†No treadmill exercise induced angina at $3 \mathrm{~mm} \mathrm{ST} \mathrm{segment} \mathrm{depression.}$

$\ddagger$ Earlier than three months before starting study treatment.

\section{Methods}

PATIENT SELECTION

The study protocol was approved by the hospital ethics committee of both centres. Ambulatory male patients of 50 to 75 years of age with stable effort angina class 2 or 3, New York Heart Association (NYHA) classification, ${ }^{10}$ treated with $180 \mathrm{mg}$ diltiazem a day and sublingual glyceryl trinitrate alone, were identified and followed up for a run in period of 15 days. Exclusion criteria were conduction defects, left ventricular hypertrophy, or a history of myocardial infarction in the previous three months. At the end of the run in period, patients who developed unstable angina, myocardial infarction, or who required additional treatment with long acting nitrates, $\beta$ blockers, calcium entry blockers, digoxin, or vasodilators were also excluded.

The remaining patients, continuing on antianginal treatment with diltiazem $180 \mathrm{mg}$ a day and glyceryl trinitrate alone, were assessed by a maximum symptom limited computerised treadmill test according to the Bruce protocol. ${ }^{11}$ A history of anginal attacks and sublingual glyceryl trinitrate consumption during the run in period was obtained, and venous blood samples taken for routine biochemical tests. Patients with a positive exercise test

Table 2 Reasons for withdrawal of patients from study, according to study group

\begin{tabular}{|c|c|c|c|c|}
\hline \multirow[b]{2}{*}{ Reasons } & \multicolumn{2}{|c|}{ Trimetazidine and diltiazem } & \multicolumn{2}{|c|}{ Placebo and diltiazem } \\
\hline & No of patients & Mean No of days* & No of patients & Mean No of days* \\
\hline Lack of efficacy & 0 & - & 2 & 8.0 \\
\hline Adverse reactions $\dagger$ & 0 & - & 2 & 7.0 \\
\hline Intercurrent illness $\ddagger$ & 1 & 27.0 & 2 & 27.0 \\
\hline Refusal to continue & 2 & 5.5 & 2 & 6.0 \\
\hline Total patients & 3 & - & 8 & - \\
\hline
\end{tabular}

* After start of study treatment.

† In the placebo group, one patient withdrew because of palpitations, anxiety, and restlessness; and the other because of constipation.

$\ddagger$ Denotes one case of fever in the trimetazidine group; and two patients reporting sick in the placebo group who could not be followed up. (defined as anginal pain plus ST segment depression of $\geqslant 1 \mathrm{~mm}$, horizontal or down sloping $80 \mathrm{~ms}$ beyond the J point, or ST segment depression of $\geqslant 3 \mathrm{~mm}$ without chest pain), and no evidence of renal or hepatic insufficiency were eligible for the study.

TREATMENT GROUPS AND MONITORING

After giving their written informed consent, patients were randomly assigned to receive either diltiazem $180 \mathrm{mg}$ and trimetazidine 60 $\mathrm{mg}$, or diltiazem $180 \mathrm{mg}$ and placebo, each day. A separate randomisation list was prepared for each centre. Gelatin capsules containing $60 \mathrm{mg}$ diltiazem plus $20 \mathrm{mg}$ trimetazidine or placebo (prepared by Serdia Pharmaceuticals, Bombay, India) were identical in taste and appearance. Patients were instructed to take one capsule after breakfast, lunch, and dinner. They were allowed sublingual glyceryl trinitrate $0.5 \mathrm{mg}$ for anginal attacks. An anginal diary was given to each patient to record daily frequency of anginal attacks and glyceryl trinitrate tablet consumption.

ASSESSMENTS AND SIDE EFFECTS

After 28 days' treatment, patients were reassessed by a maximum symptom limited computerised treadmill exercise test, according to the Bruce protocol, ${ }^{11}$ at the same time of the day. Exercise was stopped if ST segment depression of $1 \mathrm{~mm}$ with chest pain or $3 \mathrm{~mm}$ without chest pain occurred, the predicted maximum heart rate was reached, or in the event of arrhythmia, hypotension, dyspnoea, or fatigue. Venous blood samples were collected for routine biochemical tests, and the anginal diaries were retrieved from the patients. The following clinical, exercise, and biochemical variables were assessed at baseline and after 28 days' treatment: number of anginal attacks and glyceryl trinitrate tablets consumed each week; heart rate, blood pressure, and rate-pressure product at rest and exercise; times to onset of angina, $1 \mathrm{~mm}$ ST segment depression (horizontal or down sloping $80 \mathrm{~ms}$ beyond J point), and peak exercise; maximum work and maximum ST segment depression at peak exercise; total cholesterol, high density lipoprotein (HDL) cholesterol, total triglyceride, fasting blood glucose, and serum creatinine. Concurrent use of other drugs was recorded at baseline and on day 28. Side effects were monitored by asking the patient open ended questions to identify any problems that had occurred since the previous visit.

STATISTICAL ANALYSIS

Sample size was calculated to detect an increase in the number of patients responding to trimetazidine with respect to time to $1 \mathrm{~mm}$ ST segment depression, taken as the primary outcome, of at least $40 \%$, that is, from $15 \%$ (estimated increase in the placebo group) to $55 \%(\alpha=0.05, \beta=0.01)$. Response to treatment was defined as an improvement of $\geqslant 25 \%$ from baseline in clinical and stress test outcomes. Allowing for a $15 \%$ dropout rate, we calculated that 32 patients would be needed for each group. 
Table 3 Patients with stable angina showing an improvement of at least $25 \%$ from baseline, after 28 days' treatment, in clinical and exercise outcomes, according to study group

\begin{tabular}{|c|c|c|c|c|}
\hline Outcome & $\begin{array}{l}\text { Trimetazidine and } \\
\text { diltiazem }(n=32) \\
n(\%)\end{array}$ & $\begin{array}{l}\text { Placebo and } \\
\text { diltiazem }(n=32) \\
n(\%)\end{array}$ & $\begin{array}{l}\text { Difference in response \% } \\
(95 \% \mathrm{CI})\end{array}$ & $p$ value \\
\hline Anginal attacks per week & $28(87.5)$ & $15(46.9)$ & $40.6(19.9$ to 61.3$)$ & $<0.001$ \\
\hline Glyceryl trinitrate tablets per week & $25(78.1)$ & $15(46.9)$ & $31.2(8.9$ to 53.5$)$ & 0.01 \\
\hline \multicolumn{5}{|l|}{ Exercise time } \\
\hline At onset of angina & $12(37.5)$ & $5(15.6)$ & $21.9(0.9$ to 42.9$)$ & $<0.05$ \\
\hline At $1 \mathrm{~mm} \mathrm{ST} \mathrm{segment} \mathrm{depression}$ & $21(65.6)$ & $9(28.1)$ & $37.5(14.9$ to 60.1$)$ & 0.003 \\
\hline At peak exercise & $18(56.3)$ & $10(31.3)$ & $25.0(1.5$ to 48.5$)$ & 0.04 \\
\hline Maximum work at peak exercise & $17(53.1)$ & $8(25)$ & $28.1(5.2$ to 51.0$)$ & 0.02 \\
\hline $\begin{array}{l}\text { Maximum ST segment depression at peak } \\
\text { exercise }\end{array}$ & $11(34.4)$ & $10(31.3)$ & 3.13 & NS \\
\hline
\end{tabular}

CI, confidence interval.

Response to treatment was analysed on an intention to treat basis, and the categorical data compared by the standard error of difference in proportions. Change in continuous variables was analysed using data on those who completed the study, and compared by $t$ test. Significance was defined as a two tailed $\mathrm{p}$ value of $<0.05$.

Results

Sixty four eligible patients were randomised to the two treatment groups. The baseline characteristics of the patients are shown in table 1, and were balanced between the groups. The majority of patients had moderate functional
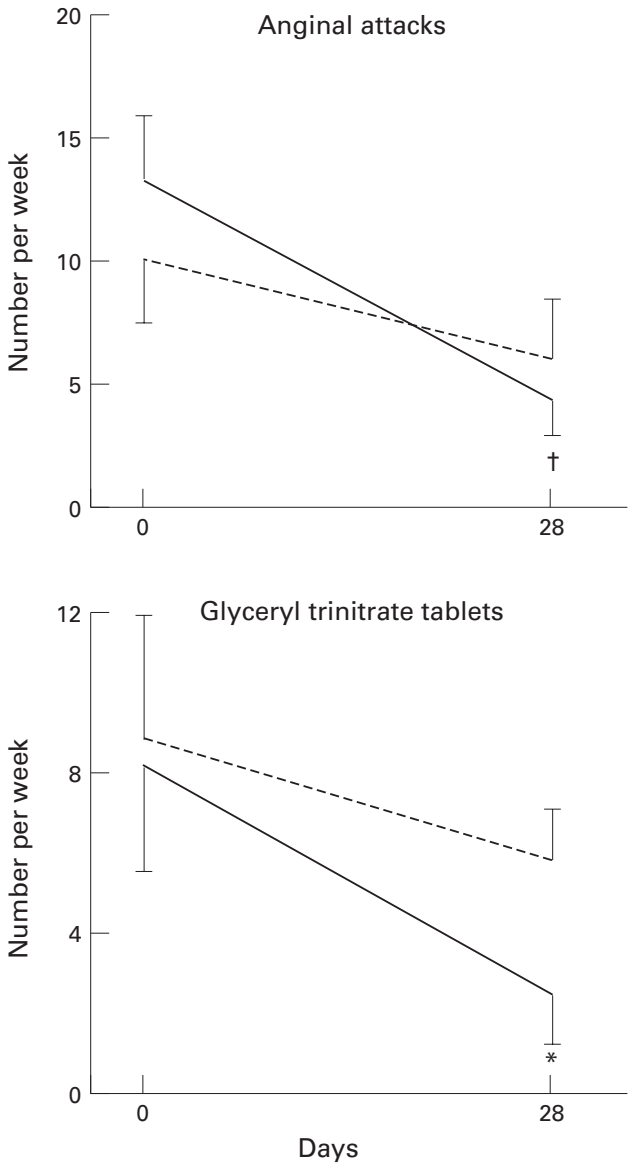

Figure 1 Mean anginal attacks and glyceryl trinitrate consumption according to study group:- diltiazem + trimetazidine, - - - diltiazem + placebo. Bars represent $95 \%$ confidence intervals. ${ }^{*} p<0.05,+p<0.01$, difference in change from day 0 after treatment between the two groups. cardiac disability, and in $12(19 \%)$ the duration of stable angina was more than five years. Nearly half the patients had silent ischaemia, there being no treadmill exercise induced angina at $3 \mathrm{~mm}$ ST segment depression. Among those recruited, 53 patients (29 of the 32 in the trimetazidine group, and 24 of the 32 in the placebo group) completed the 28 days of study medication. Three patients in the trimetazidine group, and eight in the placebo group were withdrawn from the study treatment before 28 days'. The reasons for this and the timing are shown in table 2 . Adverse events not leading to withdrawal included constipation (two patients in the trimetazidine group, compared to one in the placebo group), increased appetite in one patient in the trimetazidine group, and depression and bradycardia each in one patient in the placebo group.

The number patients in each group who met the criteria of response to treatment (defined as an improvement of at least $25 \%$ from baseline in the variable after 28 days' treatment) is summarised in table 3 . The intention to treat analysis showed significant difference between treatment groups in clinical and exercise outcomes. As compared to those in the placebo group, the number of patients with a response in the trimetazidine group was greater for anginal attacks per week by $40.6 \%$ (95\% confidence interval (CI) 19.9 to 61.3 ; p < 0.001), and for glyceryl trinitrate tablet consumption per week by $31.2 \%$ (95\% CI 8.9 to $53.5 ; \mathrm{p}=$ 0.01 ); greater for exercise time to onset of angina by $21.9 \%(95 \%$ CI 0.9 to $42.9 ; \mathrm{p}=$ $0.05)$, for exercise time at $1 \mathrm{~mm}$ ST segment depression by $37.5 \%$ (95\% CI 14.9 to 60.1 ; $\mathrm{p}=0.003$ ), and for peak exercise time by $25 \%$ ( $95 \%$ CI 1.5 to $48.5 ; \mathrm{p}=0.04)$; and greater for maximum work at peak exercise by $28.1 \%$ (95\% CI 5.2 to $51.0 ; \mathrm{p}=0.02$ ). After four weeks' treatment, the number of patients with no exercise induced angina at $3 \mathrm{~mm} \mathrm{ST}$ segment depression was 13 of 29 with trimetazidine, and 13 of 24 with placebo. The difference was not significant.

The effect of treatment in clinical outcomes is shown in fig 1 . As compared to patients in the placebo group, those in the trimetazidine group had a statistically significant improvement in the mean number of anginal attacks of 4.8 per week $(95 \%$ CI 2.1 to 7.5 ; $p<0.002)$, and in mean glyceryl trinitrate tablet consumption of 2.6 per week ( $95 \%$ CI 0.4 to 4.8 ; 

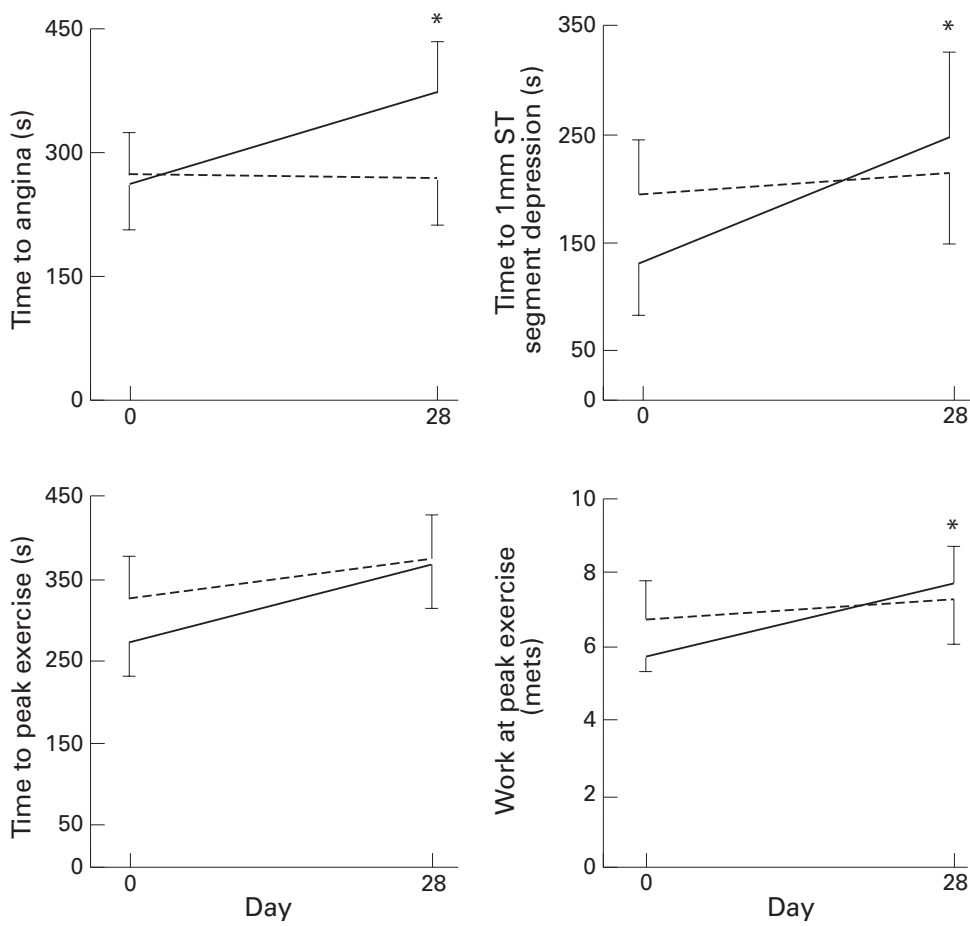

Figure 2 Mean treadmill exercise outcomes according to study group. —_ diltiazem + trimetazidine, - - diltiazem + placebo. Bars represent 95\% confidence intervals. Mets, metabolic equivalents. ${ }^{\star} p<0.05$, difference in change from day 0 after treatment between the two groups. cise time to onset of angina, exercise time at 1 $\mathrm{mm}$ ST segment depression, and maximum work attained at peak exercise.

Although a significantly greater number of patients benefited in exercise time to peak exercise (table 3), by about $50 \mathrm{~s}$ (fig 2), this treatment effect did not reach statistical significance. A recent controlled study on combination treatment with trimetazidine and diltiazem has confirmed some of these treatment effects. ${ }^{12}$ The lack of an effect of the addition of trimetazidine to diltiazem on resting and exercise heart rate and blood pressure is consistent with the findings in studies with trimetazidine alone, ${ }^{7}$ and its mechanism of action. ${ }^{6}$

The conventional treatment of stable angina is with nitrates, $\beta$ blockers, and calcium entry blockers, and they exert their influence by acting on the determinants of the myocardial oxygen supply-demand balance. ${ }^{1}$ Despite their therapeutic efficacy, there are problems with adverse effects, or contraindications to their use, and the fact that they are not universally effective when used alone or in combination. ${ }^{1}$ In a placebo controlled study of combination treatment with these agents (nifedipine, isosorbide mononitrate, or both drugs in combination with atenolol) there was no advantage over monotherapy in patients with stable angina. ${ }^{2}$ Favourable experience of combination therapy with diltiazem, a calcium entry blocker, and trimetazidine in patients with stable angina encouraged us to study these two drugs.

Diltiazem is effective in controlling symptoms of stable angina, ${ }^{13}$ and trimetazidine as a single agent has been shown in controlled studies to have an antianginal efficacy greater than placebo, ${ }^{7}$ and equal to nifedipine ${ }^{8}$ or propranolol. ${ }^{9}$ The two drugs work through different mechanisms. Diltiazem increases coronary blood flow by direct coronary artery vasodilatation, and reduces myocardial oxygen demand by peripheral arterial vasodilatation and a negative inotropic effect. ${ }^{1}$ Trimetazidine may act by influencing and partly correcting the metabolic consequences of ischaemia and reperfusion in the myocardium. ${ }^{6}$ Increasingly, evidence has focused attention on the cellular metabolic consequences of ischaemia, ${ }^{14}$ which, at mitochondrial level, results in a fall in energy to the cytoplasm, depletion of the high energy phosphate compounds ATP and phosphocreatine, and accelerated glycolysis, glycogenolysis, and lactate production. ${ }^{15}$ The poor washout causes an accumulation of protons. ${ }^{16}$ On reperfusion, the exacerbation of ischaemic injury by toxic free radical species is well established. ${ }^{17}$ Trimetazidine has been shown to increase creatine rephosphorylation, increase ATP and phosphocreatine concentrations during reperfusion, and to act as an intracellular buffer to lessen the fall in $\mathrm{pH}$, in the isolated perfused rat heart rendered ischaemic. ${ }^{18}$ In the same model, the free radical scavenging effect of trimetazidine has been demonstrated directly, ${ }^{19}$ and in red cells treated with free radical generating agents, it reduced malondialdehyde production, a marker of lipid peroxidation by free radicals. $^{20}$ The direct cellular action of 
trimetazidine is supported by evidence in humans during CABG and PTCA. Oral pretreatment of patients with trimetazidine, and its addition to the cardioplegic solution during CABG, significantly reduced malondialdehyde levels in the coronary sinus, and improved left ventricular function compared to placebo, ${ }^{21}$ and intracoronary injection of trimetazidine during PTCA significantly delayed ST segment shift, decreased maximum ST segment shift, and reduced maximum $\mathrm{T}$ wave changes compared to placebo, without altering heart rate or intracoronary and systemic pressures. $^{22}$ The intracellular metabolic mode of action of trimetazidine may also be clinically useful in chronic heart failure. In a small controlled study of six months' duration on patients with NYHA class 3 and 4 ischaemic cardiomyopathy, stabilised on long acting nitrates, digitalis, diuretics, oral anticoagulants, and antiarrhythmic drugs, trimetazidine significantly reduced dyspnoea and cardiac volume, and increased ejection fraction compared to placebo. $^{23}$

Our protocol in which a combination of diltiazem and trimetazidine was compared with diltiazem plus placebo provided a statistically efficient way of evaluating the short term efficacy and safety of the combination. The adverse events were similar to those associated with diltiazem, ${ }^{13}$ and their frequency was not increased by the addition of trimetazidine. Unacceptable haemodynamic side effects such as reflex tachycardia, hypotension, bradycardia, fatigue, and overt left ventricular failure or atrioventricular block found with other drug combinations (such as $\beta$ blockers with calcium entry blockers or nitrates) ${ }^{13}$ did not occur in this trial. The addition of trimetazidine to diltiazem did not affect renal function, blood glucose, or plasma lipids.

The value of the type of combination treatment we studied is that symptoms and exercise stress test outcomes in patients with stable angina improved to a clinically important degree, with minimal side effects. By combining a coronary vasodilator such as diltiazem with trimetazidine, which may correct the cellular metabolic consequences of ischaemia, the combination offers an alternative approach to the management of stable angina. Long term follow up of patients treated with this combination are needed to assess whether the benefits are maintained, and whether long term adverse effects are increased.

We are indebted to Dr David Park, consultant epidemiologist; Dr Jayalakshmi Aiyengar, Dr Prashant Desai, and Dr Niti Dube for assistance in organising the study; Mrs C Almeida for secre- tarial assistance; and Dr Prakash Kamat and Dr K A Sambasivam who helped in data collection. The study was supported by financial grant to each institution, and supplies of the herapeutic medications used, by Serdia Pharmaceuticals (India) Ltd.

1 Lazar EJ, Frishman WH. Profile of an ideal antianginal agent. Drugs 1989;38(suppl 2):1-8S.

2 Akhras F, Jackson G. Efficacy of nifedipine and isosorbide mononitrate in combination with atenolol in stable angina. Lancet 1991;338:1036-9.

3 McMurray J, Rankin A. Recent advances in cardiology-1: treatment of myocardial infarction, unstable angina, and angina pectoris. BMF 1994;309:1343-51.

4 Rao HRG, White JG. Coronary artery disease: an overview of risk factors. Indian Heart 7 1993;45:143-53. 5 Robertson RM, Robertson D. Drugs used for the treatment
of myocardial ischaemia. In: Hardman IG, Limbird LE, eds. Goodman and Gilman's the pharmacological basis of therapeutics, 9th ed. New York: McGraw-Hill, 1996:775-6.

6 Harpey C, Clauser P, Labrid C, Fryria J, Poirier J. Trimetazidine, a cellular anti-ischaemic agent. Cardiovasc Drugs Rev 1989;6:292-312.

7 Sellier P, Audouin P, Payen B, Corona P, Duong TC, Ourbak $P$. Acute effects of trimetazidine evaluated by exercise testing. Eur f Clin Pharmacol 1987;33:205-7.

8 Dalla-Volta S, Maraglino G, Della-Valentina P, Viena P, Desideri A. Comparison of trimetazidine with nifedipine in effort angina: a double-blind crossover study. Cardiovasc Drugs Ther 1990;14(suppl 4):853-9.

9 Detry JM, Sellier P, Pennaforte S, Cokkinos D, Mathes P. Trimetazidine: a new concept in the treatment of angina. Comparison with propranolol in patients with stable angina. Br f Clin Pharmacol 1994;37:279-88.

10 Braunwald E. Examination of the patient, part 1 -the history. In: Braunwald E, ed. Heart disease, a textbook of cardiovascular medicine, 3rd ed. Philadelphia: WB Saunders, 1988;12.

11 Bruce RA, Blackmon JR, Jones JW, Strait G. Exercise testing in adult normal subjects and cardiac patients. Pediatrics 1963;32:742-5

12 Levy S, and group of South of France investigators. Combination therapy of trimetazidine with diltiazem in patients with coronary artery disease. In: Maseri A, ed. From mitochondrial metabolism to coronary artery disease: new trends in management of myocardial ischaemia. Am f Cardiol 1995;76:8-12B.

13 Garcia-Palmieri $M$. The treatment of stable angina pectoris. Postgrad Med f 1992;68(suppl 1):61-4S.

14 Opie LH. Myocardial ischaemia-metabolic pathways and implications of increased glycolysis. Cardiovasc Drugs Ther 1990;4:777-90

15 Opie LH. High energy phosphate compounds. In: RakeHolland AJ, Noble MIM, eds. Cardiac metabolism. New York: John Wiley \& Sons, 1983:279-307.

16 Gevers W. Generation of protons by metabolic processes in heart cells. $\mathcal{F}$ Mol Cell Cardiol 1977;9:867-74.

$17 \mathrm{McC}$ ord JM. Oxygen derived free radicals in post-ischaemic tissue injury. N Engl f Med 1985;312:159-63.

18 Rossi A, Lavanchy N, Martin J. Anti-ischaemic effects of trimetazidine: P-NMR spectroscopy study in the isolated rat heart. Cardiovasc Drugs Ther 1990;4:812-3.

19 Maupoil V, Rochette L, Tabard A, Clauser P, Harpey C. Direct measurement of free radical generation in isolated rat heart by electron paramagnetic resonance spectroscopy: effect of trimetazidine. In: Emerit I, ed. Antioxidants in therapy and preventive medicine. New York: Plenum Press, 1990:373-6.

20 Maridonneau P, Harpey C. Trimetazidine protects the human red blood cell against oxygen free radical damage. Cardiovasc Drugs Ther 1990;4:818-9.

21 Fabiani MD, Ponzio O, Emerit I, Massonet-Castel S, Paris $\mathrm{M}$, Chevalier P, et al. Cardioprotective effect of trimetazidine during coronary artery graft surgery. F Cardiovasc Surg 1992;33:486-91.

22 Kober G, Buck T, Sievert H, Vallbracht C. Myocardial protection during percutaneous transluminal coronary angioplasty: effects of trimetazidine. Eur Heart $\mathcal{F}$ 1992;13: 1109-15.

23 Bricaud H, Brottier L, Barat JL, Combe C, Boussens B, Bonnet J. Cardioprotective effect of trimetazidine in severe ischaemic cardiomyopathy. Cardiovasc Drugs Ther 1990;4: $861-6$. 